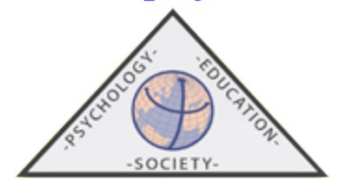

ISSN 2171-2085 (print) / ISSN 1989-709X (online)

Doi 10.25115/psye.v10i1.2485

\title{
Aprendizaje conectado en redes sociales
}

\author{
Sonia SANTOVEÑA-CASAL y Susana LÓPEZ
}

Universidad Nacional de Educación a Distancia (UNED)

(Recibido el 24 de Mayo de 2019, Aceptado el 29 de Septiembre de 2020)

RESUMEN: En el marco del proyecto Análisis de pedagogías digitales: Comunicación, Redes Sociales y nuevas narrativas ( $\mathrm{CoReN}$ ) se ponen en marcha distintas propuestas pedagógicas digitales. En este artículo se presentan los resultados obtenidos en el desarrollo de un modelo de aprendizaje conectado en redes sociales. Se llevan a cabo varios debates académicos en Twitter en el marco de distintas asignaturas. Posteriormente, se evalúan los resultados obtenidos a través del desarrollo de diferentes grupos de discusión realizados con los estudiantes. El análisis de datos es cualitativo, basado en el análisis de contenido de los grupos de discusión. Los resultados indican que los estudiantes valoran muy positivamente la puesta en marcha de propuestas innovadoras en los estudios oficiales. Se concluye que la participación de carácter académico a través de Twitter ha mejorado el proceso comunicación e interacción y también el aprendizaje de los estudiantes.

Palabras clave: Twitter; comunicación; nuevas narrativas; pedagogía digital.

\section{Learning Connected in Social Networks}

\begin{abstract}
In the framework of the project Analysis of digital pedagogies: Communication, Social networks and new narratives (CoReN), different digital pedagogical proposals are launched. In this paper the results are presented in the development of a learning model connected in social networks. Several academic debates are carried out on Twitter in the framework of different subjects. Subsequently, the results are evaluated through the development of several discussion groups carried out with the students. The data analysis is qualitative, based on the content analysis of the discussion groups. The results indicate that students value very positively the implementation of innovative proposals in official studies. It is concluded that the academic participation on Twitter has improved the process of communication and interaction and also the learning of the students.
\end{abstract}

Keywords: Twitter; Communication; New Narratives; Digital Pedagogy.

Correspondencia: Sonia Santoveña-Casal. C/ Juan del Rosal 16, 28040 Madrid. E-mail: ssantovena@ edu.uned.es 


\section{Introducción}

El aprendizaje conectado se desarrolla por medio de procesos de hiperconexión y relación, a través de los lazos interactivos que tienen lugar en una comunidad o red. Las relaciones interpersonales son la base de su desarrollo. Son clásicos los estudios que han dado importancia de los procesos de interacción y comunicación online entre el alumnado y/o con el profesorado, donde queda claro que las conexiones entre las personas constituyen un elemento fundamental en nuestra experiencia de aprendizaje (Santoveña, Navarro y Bernal, 2018; Weidlich y Bastiaens, 2017, entre otros). Autores como Luo, Zhang y Qi (2017) encontraron que interacción la estudiante-alumno y estudiante-profesor refuerza el sentido de pertenencia de los estudiantes.

Las investigaciones centradas en el aprendizaje conectado dentro del marco de las redes sociales están adquiriendo un cierto protagonismo. Aunque ya no es una novedad hablar de redes sociales en el aprendizaje, sigue siendo crucial conocer su posible influencia en los procesos de motivación y en el aprendizaje y el primer paso es analizar qué opinan los estudiantes (Lee y Bonk, 2016).

Las investigaciones en el campo de las redes sociales y el aprendizaje están siendo muy numerosa (entre otros, Mamonov, Koufaris y Benbunan-Fich, 2016; Mercer, Hennessy y Warwick, en prensa; Sobaih, Moustafa, Ghandforous, y Khan, 2016; Scott, Sorokti, y Merrell, 2016; Santoveña-Casal, 2019; Wong, Sing-Chai, y Poh-Aw, 2017). Aunque, no es posible hablar de resultados concluyentes.

Se ha encontrado que las redes constituyen potenciales espacios de aprendizaje y que pueden facilitar el mismo (Lambić, 2016; Milošević, Živković, Arsić, y Manasijević, 2015; Wong, Sing-Chai, y Poh-Aw, 2017). No solo funcionan como espacio de aprendizaje formal, sino también informal (Lu, Hao, y Jing, 2016; Wodzicki, Schwämmlein y Moskaliuk, 2012).

De hecho, se ha hallado que la motivación de los estudiantes, con relación al aprendizaje, mejora al utilizar redes sociales (Akcaoglu y Bowman, 2016; Eid y Al-Jabri, 2016; Mason y Fennie, 2008). La participación en un debate académico a través de Twitter influye en la percepción del alumnado, no solo en términos de satisfacción y utilidad, sino que, además, el alumnado considera que mejora su aprendizaje y rendimiento académico (Santoveña, Navarro y Bravo, 2018). Autores como Mason y Fennie (2008), afirman que, actualmente, el aprendizaje digital, tanto a través de recursos digitales, como de redes sociales juegan un papel relevante en el compromiso del alumnado en el aprendizaje. Las redes, además, ofrecen la posibilidad de generar comunidades (Blight, Ruppel, y Schoenbauer, 2017) y que son medio de comunicación y un espacio de colaboración que puede facilitar el aprendizaje (Tuzel y Hobbs, 2017). Todos estos aspectos pueden ser variables influyentes en la mejora motivacional que se ha observado en estudiantes que toman parte de actividades académicas desarrolladas en redes sociales.

El proyecto CoReN: Análisis de pedagogías digitales: Comunicación, Redes Sociales y nuevas narrativas se aprueba en 2018 por el Vicerrectorado de Metodología e Innovación y el apoyo del Instituto Universitario de Educación a Distancia (IUED), dentro del Plan de apoyo a la innovación docente en la UNED. Su El desarrollo de prácticas pedagógicas digitales, basadas en procesos comunicacionales y de interacción, redes sociales y nuevas narrativas, desde una perspectiva crítica y creativa, que faciliten la adquisición de competencias prácticas, de gestión 
y de pensamiento crítico, así como, que refuercen la adquisición de conocimientos relativos a las materias impartidas en los estudios, constituye su principal objetivo.

El proyecto se ha desarrollado en dos partes principales: investigación y experimentación. En primer lugar, se realiza un análisis previo de necesidades y expectativas, para posteriormente poner en marcha las iniciativas pedagógicas en las tres áreas de implementación (fase de experimentación) y en una tercera etapa se analiza la opinión de los estudiantes sobre las experiencias desarrolladas (fase de investigación).

En definitiva, el objetivo general de la investigación presentada en estas páginas es conocer analizar la efectividad de la propuesta pedagógica digital basada en un proceso de aprendizaje conectad en redes sociales, un proceso de participación social.

Los objetivos específicos son:

1. Analizar los aspectos pedagógicos considerados creativos e innovadores.

2. Conocer cómo ha influido los procesos de comunicación e interacción en el aprendizaje.

3. Analizar las dificultades encontradas en el debate académico a través de Twitter.

4. Estudiar el aprendizaje adquirido en la asignatura.

\section{Diseño y metodología}

Es un diseño cualitativo basado en el análisis de contenido de las respuestas aportadas por los estudiantes en los grupos de discusión que tenían como objetivo valorar la propuesta pedagógica digital.

La investigación cualitativa tiene como objetivo acercarnos a la realidad exterior, ayuda a conocer las experiencias (individuales o grupales, las interacciones y comunicaciones) en su contexto natural y detectar las individualidades en estas experiencias, teniendo en especial consideración el contexto, basándose gran parte de las ocasiones en estudios de caso (Flick, 2012). En este artículo, el contexto natural de la experiencia es digital, Twitter y el estudio de caso son los grupos de estudiantes analizados. El enfoque cualitativo es la herramienta ideal para comprender la experiencia de aprendizaje desde la perspectiva grupal del alumnado.

El análisis de contenido se desarrolla siguiendo las orientaciones realizadas por Rapley (2014). En primer lugar, para llevar a cabo el proceso de codificación se escuchó en varias ocasiones el grupo de discusión. Se tomó notas de aquello considerado más general, así como lo ideas interesantes, singular y raro. En segundo lugar, se aplican códigos a estas secciones más específicas. En tercer lugar, se realizan comparaciones dentro de los temas que han salido en las conversaciones, teniendo en cuenta los ejemplos que producen 'desviaciones' que hacen repensar el análisis. Por tanto, para llevar a cabo esta codificación y categorización se realizan comparaciones constantes, se buscan aquellas partes del discurso más relevantes y se analizan comparándolas entre las distintas opiniones, para finalmente denominarlas y codificarlas, como indican Flick (2015) y Gibbs (2012). Finalmente, se recogen las ideas consideradas de mayor relevancia y valor. Se extraen categorías generales y la relación entre estas categorías, formando un mapa conceptual de las opiniones.

En síntesis, el análisis realizado con las opiniones de los estudiantes ha implicado, como en la mayoría de las investigaciones cualitativas, gestionar toda la información y posteriormente 
interpretarla. Dos aspectos considerados comunes en el análisis de datos cualitativos (Coffey y Atkinson, 1996; Mason, 2002).

El mapa conceptual se realiza con el apoyo del software Atlas.ti HM que facilita la representación de las cuatro familias obtenidas (Innovación y creatividad, Aprendizaje, Comunicación

y Dificultad) todas dependientes de la superfamilia "Debate académico en Twitter (Grupos de discusión).

Los comentarios de los estudiantes se codifican según el número de grupo 1, 2 o 3 y el orden de intervención.

El diseño del protocolo del grupo de discusión se realizó en el marco del proyecto con el apoyo de un grupo de profesores. Se establecen tres bloques de contenidos:

1. Valoración general de la propuesta pedagógica: ¿Qué aspectos concretos de la asignatura destacaría como creativos y valiosos?

2. Proceso de comunicación: ¿Cómo le han influido los procesos de comunicación e interacción en el proceso de aprendizaje?

3. Proceso de aprendizaje

- ¿Qué tipo de aprendizaje considera que ha adquirido?

- ¿Dificultades encontradas?

Se llevaron a cabo tres grupos de discusión. Uno de ellos formado por estudiantes de distintas asignaturas (Grado y Posgrado) y otros dos grupos formados por estudiantes de la misma asignatura de posgrado. Los grupos se desarrollan a través de Skipe o de webconferencia por medio de los cursos virtuales de las asignaturas.

\section{Contexto de aplicación}

En el marco de distintas asignaturas (3) de Grado y Posgrado se llevan a cabo debates académicos a través de Twitter como actividad de aprendizaje conectado y participación social. Las características de la actividad propuesta es la misma para las tres asignaturas, lo que permite realizar un estudio conjunto sobre las valoraciones del alumnado sobre la propuesta pedagógica y su efectividad. La diferencia entre las tres asignaturas es el contenido del debate, puesto que los debates son adaptados a los programas docentes de cada una de ellas.

Los debates comienzan con el envío por parte del profesorado de un mensaje en twitter; sin embargo, los estudiantes pueden consultar en la guía didáctica los temas y preguntas de discusión, así como el calendario del mismo. Se desarrollan a lo largo de tres semanas. Cada semana se inicia un debate diferente.

\section{Resultados}

Los estudiantes han valorado la posibilidad de tener una 'interacción muy directa' (G2_1), que la actividad les haya facilitado la toma de contacto con otros estudiantes, aspecto especialmente importante porque, como comenta G2_2: '( ...) como estamos en la UNED es difícil tener comunicación con los estudiantes'. Otro de los aspectos destacados por los estudiantes ha sido el tipo de comunicación ágil y rápida que se ha mantenido con el equipo docente no solo por Twitter, sino también por correo electrónico. Han subrayado cómo a través de la comunicación en Twitter 
'llegas a ver el aspecto emocional' de los otros estudiantes (G3_1), señalando que lo más interesante es ver cómo otros expresan sus opiniones (G3_4) y empatizan contigo [se dieron cuenta que estaba preocupada (...) y me sentí apoyada (...), es muy positivo. (...) Es verdad que surgen situaciones tensas (...) el caso es acércanos cuando estamos lejos' (G3_3)]. Se han sentido apoyado por otros estudiantes, así como por el profesorado.

Se ha observado que los estudiantes valoran la valoración de la propuesta pedagógica, destacando la revelancia de desarrollar e implementar pedagogías innovadoras y creativas como ha sido la experiencia de Twitter y puesta en marcha en la asignatura. Consideran que las redes sociales '(...) han supuesto toda una revolución' (G1_1), valorando este tipo de experiencias 'fundamental para futuros profesores' (G1_1).

La conexión de los contenidos de la asignatura con los debates propuestos ha requerido del alumnado una lectura y proceso de documentación previo antes de practicar. Por esta razón, la participación en el debate ha ayudado a la adquisición de conocimento y al aprendizaje. Así lo han comentado en los grupos de discusión. Además, unos de los requisitos de evaluación de la participación era la relevancia de los comentarios enviados. Como dice G2_2 '(...) hacer los debates implicaba que tenía que buscar información sobre lo que había que hablar (...) No tenía tanto conocimiento y (...) tenía que buscar noticias y ha sido una forma de aprender de manera autónoma (...). De hecho, han propuesto poder extender este tipo de actividades al resto de los temas de la asignatura y dedicar, por ejemplo, 'tres días específicos para un tema (concreto) a través de Tw' (G3_1). Por otra parte, han valorado muy positivamente el proceso de aprendizaje reflexivo llevado a cabo, puesto que, como comenta G2_2, era necesario no solo 'defender tu postura (...)', sino también se requería '(...) saber cambiar de opinión en función de la opinión de otros'.

La propuesta no está exenta de dificultades. Por un lado, algunos estudiantes han encontrado problemas técnicos y psicológicos al enfrentarse con una actividad nueva. En segundo lugar, han destacado dificultades relacionadas con el tipo de discurso y características de la red social. Y, finalmente, consideran que han existido problemas con el debate en sí mismo. Las principales dificultades destacas han sido las relativas a la hora de iniciarse con una actividad nueva y la barrera que supone 'enfrentarse a algo nuevo' (G1_2), pero valorando que una vez inicada, te formas y ves la utilidad (G1_2). Comentan que esta actividad ha supuesto su primer contacto con las redes sociales y que como adultos, padres y trabajadores ha requerido un esfuerzo extra y un verdadero reto. Es interesantes destacar cómo al principio, antes de ponerse a trabajar con Twitter, la propuesta pedagógica les pareció muy negativa ['Cuando vi que tenía un trabajo en TW para mí fue un punto negativo (...) pensé, me voy a perder dentro de Twitter' G1_3] pero, una vez, realizada la primera inversión en Twitter y comenzado a interactuar en la red, la experiencia llegó a ser muy positiva ['Decidí que ¡a ello! Vi que no era complicado, que no perdía (...) Miré el tutorial y antes de terminarlo, no volví al tutorial y logré los con seguidores (...) Paso de ser una actividad muy productiva donde me lo pasaba bien, donde leí muchísimo, me encontré con recursos impresionante' (G1_3)].

Por otra parte, los estudiantes destacan como dificultad la necesidad de adaptar el tipo de discurso a las características técnicas de la red; no solo por el número de caracteres, sino, también, por tener que integrar el un hashtag específico que define la asignatura y el tipo de debate con el que está relacionado el tweet enviado. El estudiante G3_4 lo resume muy bien cuando 
comenta que: 'escribía, pero no ponía el hashtag. Eso lo más difícil. Es un arma de doble filo, pero me ha ayudado a resumir y expresarme. He aprendido de los compañeros o el retuitear (...) La capacidad de síntesis era complicado para mí (...)'.

Como tercera dificultad han destacado la saturación de información y de mensajes que supone la participación en Twitter y el alto número de estudiantes participando en el mismo. Como comenta G2_1, en los debates contenían demasiados comentarios repetidos, en los que los estudiantes no solían leer los comentarios realizados con anterioridad por otros compañeros, lo que supuso para G2_1 '(...) tener que responder al mismo comentarios varias veces', con la sensación de no avanzar en la discusión y de estar manteniendo pequeñas conversaciones a pares, más que un debate en grupo. La rapidez del debate y las dificultades para hacer el seguimiento de todas las opiniones es una de las limitaciones comentadas. Se ha comentado que a través de Twitter se han encontrado situaciones donde '( ...) se lanzan ideas que en cara a cara no se admiten' y la necesidad de aprender a 'gestionar las cosas negativas'.

Se presenta el mapa conceptual resultado de los grupos de discusión (Figura 1):

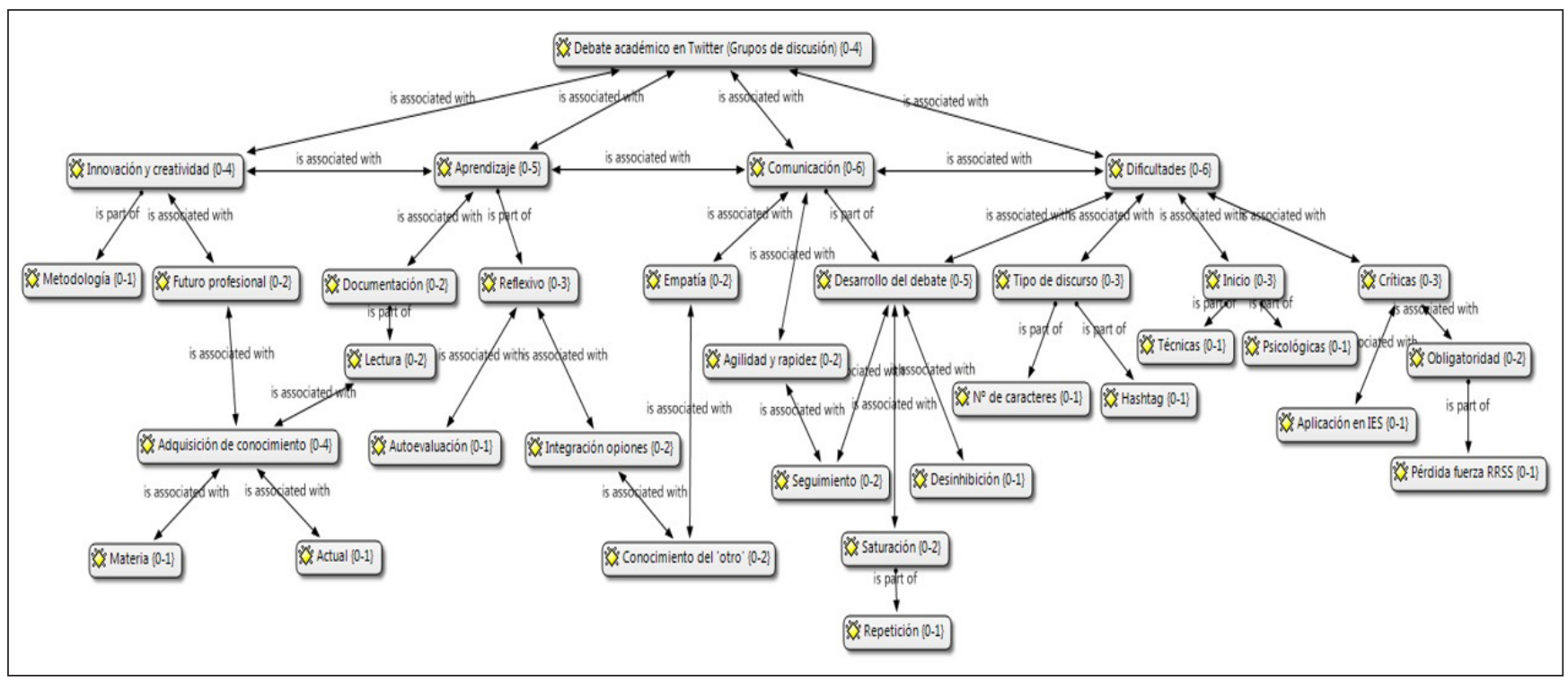

Figura 1

\section{Conclusiones}

Se concluye que la experiencia pedagógica digital ha sido positivamente evaluado por el alumnado. Por una parte valoran que el profesorado tenga interés en desarrollar propuestas arriesgadas e innovadoras que permitan adquirir compentencias relevantes de participación social y aprendizaje conectado a los estudiantes. La participación en actividades creativas, a pesar el esfuerzo añadido que suponen, son valoradas muy positivamente por los estudiantes. Reconocen que a pesar de las resistencias y expectativas negativas que tenían al comienzo de la actividad, la experiencia ha sido positiva y que la iniciativa les ha permitido desarrollar competencias útiles para su futuro profesional. En esta misma línea, son los estudios realizados por Santoveña, Navarro y Bravo (2018) que constataron que el alumnado cuando participa en debates académicos en redes 
como Twitter su percepción de la experiencia didáctica mejoraba significativamente en aspectos como la satisfacción, utilidad y el aprendizaje adquirido.

Como hemos visto, la participación social a través de Twitter no está exento de dificultades: técnicas, psicológicas como es la ansiedad que genera la intensidad de los debates, así como el enfrentarse a una actividad nueva o el tipo de discurso que requiere Twitter (rápido, breve, efímero). Sin embargo, se concluye que este tipo de actividades tiene un elemento motivacional del que carecen otras actividades más clásicas. Al igual que Akcaoglu y Bowman (2016) y Eid y Al-Jabri (2016) se observa que la motivación de los estudiantes, con relación al aprendizaje, mejora al utilizar redes sociales. Por tanto, parece que, como han indicado Mason y Fennie (2008), los recursos digitales que favorezcan la inteacción como las redes pueden jugar un papel protagonista en el refuerzo del compromiso del alumnado.

En síntesis, si el aprendizaje conectado se desarrolla sobre la base de procesos de hiperconexión y relación, a través de los lazos interactivos, está claro que en el aprendizaje conectado, los procesos de interacción y comunicación entre el alumnado y/o con el profesorado son esenciales, como han encontrado Santoveña, Navarro y Bernal (2018) y Weidlich y Bastiaens (2017). Estas propuestas pedagógicas tienen un valor añadido relacionado sobre todo por reforzar procesos de comunicación e interacción entre los estudiantes en un entorno más informal pero con un objetivo formativo. El intercambio de puntos de vista, la comunicación ágil y rápida, la adquisición de conocimiento, el desarrollo de un proceso de aprendizaje reflexivo durante los debates y posteriormente en la autoevaluación han sido aspectos muy valorados.

\section{Agradecimientos}

Esta investigación se ha realizado en el marco del Proyecto CoReN, Análisis de pedagogías digitales: Comunicación, Redes Sociales y Nuevas Narrativas. Proyecto financiado por el Vicerrectorado de Metodología e Innovación en apoyo del Instituto Universitario de Educación a Distancia (IUED), dentro del Plan de apoyo a la innovación docente en la UNED. Comenzó en enero de 2018 y finaliza en enero de 2019, con una duración de 12 meses.

\section{Referencias}

Akcaoglu, M., y Bowman, ND. (2016). Using instructor-led Facebook groups to enhance students' perceptions of course content. Computers in Human Behavior, 65: 582-590.

Coffey, A. y Atkinson, P. (1996). Making sense of qualitative data analysis: Complementary Research Strategies. Londres: Sage.

Eid, MIM., y Al-Jabri, IM. (2016). Social networking, knowledge sharing, and student learning: The case of university students. Computers \& Education, 99, 14-27.

Flick, U. (2012). Introducción editorial, en Graham Gibbs (2012), El análisis de datos cualitativos en investigación cualitativa. Madrid: Morata.

Flick, U. (2015). El diseño de investigación cualitativa. Madrid: Morata.

Gibbs, G. (2012). El análisis de datos cualitativos en investigación cualitativa. Madrid: Morata. Lambić, D. (2016). Correlation between Facebook use for educational purposes and academic performance of students. Computers in Human Behavior, 61, 313-320. 
Lee J., y Bonk, CJ. (2016). Social network analysis of peer relationships and online interactions in a blended class using blogs. The Internet and Higher Education, 28, 35-44.

Lu, J., Hao, Q, y Jing, M. (2016). Consuming, sharing, and creating content: How young students use new social media in and outside school. Computers in Human Behavior, 64, 55-64.

Luo, N., Zhang, M., y Qi, D. (2017). Effects of different interactions on students' sense of community in e-learning environment, Computers \& Education, 115: 153-160.

Mamonov, S., Koufaris, M. y Benbunan-Fich, R. (2016). The role of the sense of community in the sustainability of social network sites. International Journal of Electronic Commerce, 470-498

Mason, J. (2002). Qualitative Researching. Londres: Sage.

Mason, R. y Rennie, F. (2008). E-Learning and Social Networking Handbook. London, UK: Routledge.

Mercer, N., Hennessy, S. y Warwick, P. (en prensa). Dialogue, thinking together and digital technology in the classroom: Some educational implications of a continuing line of inquiry, International Journal of Educational Research.

Milošević, I, Živković, D, Arsić, S, Manasijević, D. (2015). Facebook as virtual classroom Social networking in learning and teaching among Serbian students. Telematics and Informatics, 32 (4), 576-585.

Rapley, T. (2014). Los análisis de la conversación, del discurso y de documentos en Investigación Cualitativa. Madrid: Morata.

Santoveña-Casal, S. (2019). The Impact of Social Media Participation on Academic Performance in Undergraduate and Postgraduate Students. International Review of Research in Open and Distributed Learning, 20 (1),

Santoveña, S., Navarro, C. y Bernal, C. (2018). Investigación en metodologías virtuales, redes sociales y comunicación. Barcelona: Octaedro.

Scott, KS., Sorokti, KH., Merrell, JD. (2016). Learning "beyond the classroom" within an enterprise social network system. The Internet and Higher Education, 29: 75-90.

Sobaih, AEE., Moustafa, MA., Ghandforous, P., Khan, M. (2016). To use or not to use? Social media in higher education in developing countries. Computers in Human Behavior; 2016 , 58: 296-305.

Tuzel, S., y Hobbs, R. (2017). El uso de las redes sociales y la cultura popular para una mejor comprensión intercultural. Comunicar, 51, 63-72.

Weidlich, J. y Bastiaens, TJ. (2017). Explaining social presence and the quality of online learning with the SIPS model. Computers in Human Behavior, 72: 479-487.

Wodzicki, K., Schwämmlein, E., y Moskaliuk, J. (2012). “Actually, I Wanted to Learn”: Studyrelated knowledge exchange on social networking sites. The Internet and Higher Education, 15 (1), 9-14.

Wong, L., Sing-Chai, C., y Poh-Aw, G. (2017). Aprendizaje de idiomas “sin costuras": Aprendizaje de segundas lenguas y redes sociales. Comunicar, 50, 9-21. 